\title{
Vertex sequences in graphs*
}

\author{
Teresa W. Haynes ${ }^{1,2, \dagger}$, Stephen T. Hedetniemi ${ }^{3}$ \\ ${ }^{1}$ Department of Mathematics and Statistics, East Tennessee State University, Johnson, TN 37614-0002, USA \\ ${ }^{2}$ Department of Mathematics and Applied Mathematics, University of Johannesburg, Auckland Park 2006, South Africa \\ ${ }^{3}$ School of Computing, Clemson University, Clemson, SC 29634, USA
}

(Received: 22 May 2020. Accepted: 25 June 2020. Published online: 11 March 2021.)

(c) 2021 the authors. This is an open access article under the CC BY (International 4.0) license (www.creativecommons.org/licenses/by/4.0/).

\begin{abstract}
We consider a variety of types of vertex sequences, which are defined in terms of a requirement that the next vertex in the sequence must meet. For example, let $S=\left(v_{1}, v_{2}, \ldots, v_{k}\right)$ be a sequence of distinct vertices in a graph $G$ such that every vertex $v_{i}$ in $S$ dominates at least one vertex in $V$ that is not dominated by any of the vertices preceding it in the sequence $S$. Such a sequence of maximal length is called a dominating sequence since the set $\left\{v_{1}, v_{2}, \ldots, v_{k}\right\}$ must be a dominating set of $G$. In this paper we survey the literature on dominating and other related sequences, and propose for future study several new types of vertex sequences, which suggest the beginning of a theory of vertex sequences in graphs.
\end{abstract}

Keywords: domination; total domination; connected domination; dominating sequences; vertex sequences; vertex cover; zero forcing number; irredundance; grid graphs.

2020 Mathematics Subject Classification: 05C69.

\section{Introduction}

We present a variety of types of vertex sequences, which are defined in terms of a requirement that the next vertex in the sequence must meet. In particular, we consider requirements that have to do with domination and related graph parameters. In Section 2, we give a description of four types of "dominating" sequences that have been defined in the literature. Then we focus on two of these sequences, namely dominating sequences and total dominating sequences. We give a brief overview as well as a few new observations concerning dominating sequences in Section 3 and a survey of known results on total dominating sequences in Section 4. In Section 5, we propose several new types of vertex sequences for future study.

We first give some terminology.

Let $G=(V, E)$ be a graph of order $|V|$ and size $|E|$. Let $S=\left(v_{1}, v_{2}, \ldots, v_{k}\right)$ be an ordered sequence of distinct vertices, and let $\widehat{S}=\left\{v_{1}, v_{2}, \ldots, v_{k}\right\}$ be the corresponding set of vertices. For ease of discussion, we let $\widehat{S}_{j}$ be the set of vertices in the first $j$ positions in $S$, that is, $\widehat{S}_{j}=\left\{v_{i} \mid 1 \leq i \leq j\right\}$.

The open neighborhood of a vertex $v \in V$ is the set $N(v)=\{u \mid u v \in E\}$, the vertices of which are called neighbors of $v$. The closed neighborhood of vertex $v$ is the set $N[v]=N(v) \cup\{v\}$. The degree $d(v)$ of $v$ is the number of neighbors of $v$, that is, $d(v)=|N(v)|$. The minimum degree among the vertices of $G$ is denoted by $\delta(G)$, while the maximum degree is denoted by $\Delta(G)$. The open neighborhood of a set $S$ is the set $N(S)=\bigcup_{v \in S} N(v)$, and its closed neighborhood is the set $N[S]=N(S) \cup S$.

The S-private neighborhood of a vertex $v \in S$ is the set $\operatorname{pn}[v, S]=N[v] \backslash(N[S \backslash\{v\}])$; vertices in this set are called private neighbors of $v$ (with respect to $S$ ).

A set $S$ of vertices is an independent set if no two vertices in $S$ are adjacent. The vertex independence number $\alpha(G)$ is the maximum cardinality of an independent set in $G$.

A set $S$ is a dominating set if every vertex in $V \backslash S=\bar{S}$ has a neighbor in $S$, that is, $N[S]=V$. A set $S$ is a total dominating set if every vertex in $V$ has a neighbor in $S$, that is, $N(S)=V$. A (total) dominating set $S$ of $G$ is minimal if no proper subset of $S$ is a (total) dominating set of $G$. The domination number $\gamma(G)$ is the minimum cardinality of a dominating set in $G$; while the upper domination number $\Gamma(G)$ is the maximum cardinality of a minimal dominating set.

*This paper is dedicated to the memory of Professor Frank Harary, on the occasion of what would have been his 100th birthday. To those of us who knew and worked with him, with more than 650 publications and nearly 300 coauthors, Frank was one of the founding fathers and a true world ambassador of graph theory.

†Corresponding author (haynes@etsu.edu). 
The total domination number $\gamma_{t}(G)$ and the upper total domination number $\Gamma_{t}(G)$ are defined similarly. Let $G[S]$ denote the subgraph induced in $G$ by the set $S \subseteq V$.

A clique of a graph $G$ is a maximal complete subgraph of $G$, and a $k$-clique is a clique with $k$ vertices.

\section{Neighborhood vertex (dominating) sequences}

In this section we consider sequences based on the open and closed neighborhoods of vertices. There are basically four types of such sequences: (i) closed neighborhood, (ii) open neighborhood, (iii) closed-open neighborhood and (iv) open-closed neighborhood.

\subsection{Closed neighborhood}

A sequence $S=\left(v_{1}, v_{2}, \ldots, v_{k}\right)$ of distinct vertices is a closed neighborhood sequence if for all $i$ with $2 \leq i \leq k$,

$$
N\left[v_{i}\right] \nsubseteq \bigcup_{j=1}^{i-1} N\left[v_{j}\right] .
$$

A vertex $v_{i}$ dominates itself and all of its neighbors, that is, $v_{i}$ dominates all of the vertices in its closed neighborhood $N\left[v_{i}\right]$. In this type of sequence, a vertex $v_{i}$ dominates at least one vertex $x$ not dominated by any vertex preceding it in the sequence, that is, for $i \in\{2,3, \ldots k\}$,

$$
N\left[v_{i}\right] \backslash N\left[\widehat{S}_{i-1}\right] \neq \emptyset .
$$

Note that the vertex $v_{i}$ itself could be this vertex $x$. We could also say that every vertex $v_{i}$ must dominate at least one previously undominated vertex. In other words, $\operatorname{pn}\left[v_{i}, \widehat{S}_{i}\right] \neq \emptyset$.

If $S$ is a closed neighborhood sequence of maximal length in $G$, then $\widehat{S}$ must be a dominating set of $G$. Therefore, $S$ is called a dominating sequence of $G$. The maximum length of a dominating sequence $S$ of a graph $G$, $|\widehat{S}|$, is the Grundy domination number of $G$, denoted by $\gamma_{g r}(G)$. A dominating sequence of length $\gamma_{g r}(G)$ is called a Grundy dominating sequence or just a $\gamma_{g r}$-sequence of $G$.

Grundy dominating sequences were introduced in 2014 by Brešar, Gologranc, Milanič, Rall, and Rizzi [11], where they noted the following.

Proposition 2.1 (Brešar et al. [11]). For any graph $G$, the minimum length of a maximal dominating sequence equals the domination number $\gamma(G)$.

Brešar et al. attribute their idea for studying these sequences to the domination game introduced by Brešar, Klavžar, and Rall in [13]. In this game two players, Dominator and Staller, alternately choose vertices so that each chosen vertex enlarges the set of vertices of $G$ dominated to that point in the game. While the aims of these players are opposing (Dominator wants to minimize the number of moves in the game, while Staller wants to maximize the number of moves), the outcome of the game is a sequence of vertices with the property that each chosen vertex dominates at least one previously undominated vertex. The game ends when no move can be made, that is, when the sequence of vertices created by the moves is a dominating sequence of $G$. We note that the best possible outcome for Dominator is a dominating sequence of length $\gamma(G)$, and the best possible outcome for Staller is a dominating sequence of length $\gamma_{g r}(G)$, that is, a Grundy dominating sequence. Of course, dominating sequences created by the domination game can have lengths between these two extremes. We give a brief overview of selected results and few new results on the Grundy domination number in Section 3.

\subsection{Open neighborhood}

A sequence $S=\left(v_{1}, v_{2}, \ldots, v_{k}\right)$ of distinct vertices is an open neighborhood sequence if for all $i$ with $2 \leq i \leq k$,

$$
N\left(v_{i}\right) \not \bigcup_{j=1}^{i-1} N\left(v_{j}\right)
$$

A vertex $v$ total (open) dominates its neighbors, that is, $v$ total dominates all of the vertices in its open neighborhood $N(v)$, but does not total dominate itself. Thus, for any graph $G$ without isolated vertices, a sequence $S=\left(v_{1}, v_{2}, \ldots, v_{k}\right)$ is an open neighborhood sequence if for every vertex $v_{i}$ where $i \in\{2,3, \ldots k\}$,

$$
N\left(v_{i}\right) \backslash N\left(\widehat{S}_{i-1}\right) \neq \emptyset .
$$


Hence, every vertex $v_{i}$ in the sequence total dominates at least one vertex that is not total dominated by any vertex preceding it in the sequence. In other words, $v_{i}$ is adjacent to at least one vertex that has no neighbor in $\widehat{S}_{i-1}$.

If $\widehat{S}$ is a total dominating set of $G$, then $S$ is called a total dominating sequence. The maximum length of a total dominating sequence of $G$ is the Grundy total domination number of $G$, denoted by $\gamma_{g r}^{t}(G)$. A total dominating sequence of $G$ of length $\gamma_{g r}^{t}(G)$ is called a Grundy total dominating sequence, or a $\gamma_{g r}^{t}$-sequence of $G$.

Total dominating sequences were introduced in 2016 by Brešar, Henning, and Rall [12], who noted that the minimum length of a total dominating sequence of a graph $G$ equals the total domination number $\gamma_{t}(G)$. As is the case with dominating sequences, total dominating sequences are associated with the total domination game introduced in 2015 by Henning, Klavzar, and Rall [25]. We give a brief overview of the known results on Grundy total dominating sequences in Section 4.

\subsection{Closed-open neighborhood}

A sequence $S=\left(v_{1}, v_{2}, \ldots, v_{k}\right)$ of distinct vertices is a closed-open neighborhood sequence if for all $i$ with $2 \leq i \leq k$,

$$
N\left[v_{i}\right] \not \bigcup_{j=1}^{i-1} N\left(v_{j}\right)
$$

Thus, a sequence $S=\left(v_{1}, v_{2}, \ldots, v_{k}\right)$ in a graph $G$ is called an closed-open neighborhood sequence if for every vertex $v_{i}$, for $i \in\{2,3, \ldots k\}$,

$$
N\left[v_{i}\right] \backslash N\left(\widehat{S}_{i-1}\right) \neq \emptyset .
$$

In other words, some vertex in $N\left[v_{i}\right]$ has no neighbor in $N\left(\widehat{S}_{i-1}\right)$. If $\widehat{S}$ is a dominating set, then $S$ is a closed-open dominating sequence.

A closed-open dominating sequence is called an $L$-sequence in reference to the close relationship these sequences have to a type of zero forcing number (zero forcing will be defined in Section 2.5). The length of a longest $L$-sequence is called the L-Grundy domination number of $G$, and is denoted by $\gamma_{g r}^{L}(G)$. This type sequence was introduced in 2017 by Brešar, Butjtás, Gologranc, Klavžar, Košmrlj, Patkós, Tuza, and Vizer [9].

\subsection{Open-closed neighborhood}

A sequence $S=\left(v_{1}, v_{2}, \ldots, v_{k}\right)$ of distinct vertices is a open-closed neighborhood sequence if for all $i$ with $2 \leq i \leq k$,

$$
N\left(v_{i}\right) \not \bigcup_{j=1}^{i-1} N\left[v_{j}\right] .
$$

Thus, a sequence $S=\left(v_{1}, v_{2}, \ldots, v_{k}\right)$ in a graph $G$ is called an open-closed neighborhood sequence if for every vertex $v_{i}$, for $i \in\{2,3, \ldots k\}$,

$$
N\left(v_{i}\right) \backslash N\left[\widehat{S}_{i-1}\right] \neq \emptyset .
$$

In other words, $v_{i}$ is adjacent to at least one vertex that is not in $N\left[\widehat{S}_{i-1}\right]$. Again, if $\widehat{S}$ is a dominating set, then $S$ is a open-closed dominating sequence.

An open-closed dominating sequence is called a $Z$-sequence, in reference to the close relationship these sequences have to the zero forcing number, as we shall see in Section 2.5. The length of a longest $Z$-sequence is called the $Z$-Grundy domination number of $G$, and is denoted by $\gamma_{g r}^{Z}(G)$. This version was also introduced by Brešar et al. [9] in 2017.

\subsection{Relationship with zero forcing numbers}

We conclude this section with a brief discussion of zero forcing numbers and their relationship to dominating sequences.

Let the vertices of a graph $G$ be colored white and blue, where $W$ denotes the set of vertices colored white, and $B$ denotes the set of vertices colored blue. If a given blue vertex $v$ has exactly one white neighbor $w$, then by the Color Changing Rule, the color of $w$ is changed to blue. A zero forcing set of $G$ is a subset $B$ of vertices such that if initially vertices in $B$ are colored blue and the remaining vertices in $\bar{B}$ are colored white, then by repeated applications of the Color Changing Rule all the vertices in $V$ are colored blue. The zero forcing number $Z(G)$ of a graph $G$ equals the minimum cardinality of a zero forcing set in $G$.

Brešar et al. established the following relationship between the $Z$-Grundy domination number and the zero forcing number.

Theorem 2.1 (Brešar et al. [9]). If $G$ is a graph of order $n$ without isolated vertices, then $\gamma_{g r}^{Z}(G)+Z(G)=n$. 
Proof Sketch. We outline the proof of Theorem 2.1 given in [9]. Without loss of generality, we can assume that $G$ is connected and of order $n$. Let $B$ be a minimum zero forcing set, that is, the set of vertices initially colored blue, and let $k=n-|B|=n-Z(G)$. Let the following two sequences appear in the color changing rule, in which all vertices become blue: $b_{1}, b_{2}, \ldots, b_{k}$ and $w_{1}, w_{2}, \ldots, w_{k}$, where $b_{i}$ is the blue vertex selected at the $i$ th step of the color changing process, and $w_{i}$ is the unique white neighbor of $b_{i}$. They show that the sequence $\left(w_{k}, w_{k-1}, \ldots, w_{1}\right)$ is a $Z$-sequence of $G$, which implies that $\gamma_{g r}^{Z}(G) \geq k=n-Z(G)$.

Conversely, let $S=\left(u_{1}, u_{2}, \ldots, u_{k}\right)$ be a maximum $Z$-sequence for $G$. They show that $X=V \backslash \widehat{S}$ is a zero forcing set. It follows then that $Z(G) \leq|X|=n-k=n-\gamma_{g r}^{Z}(G)$, and so $\gamma_{g r}^{Z}(G)+Z(G)=n$.

In 2019 Lin [27] showed that not only the $Z$-Grundy domination number but each of the four types of Grundy domination parameters mentioned in this section has a similar relationship with a zero-forcing type of parameter.

To this result we can add the following.

A vertex $v$ in a set $S \subseteq V$ is called open irredundant if $N(v) \backslash N[S \backslash\{v\}] \neq \emptyset$. The upper open irredundance number $\operatorname{OIR}(G)$ equals the maximum cardinality of an open irredundant set $S$ in $G$. Thus, if a set $\left\{v_{1}, v_{2}, \ldots, v_{k}\right\}$ is a maximum cardinality open irredundant set, then the sequence $\left(v_{1}, v_{2}, \ldots, v_{k}\right)$ is automatically an open-closed neighborhood sequence, and the following result is apparent.

Proposition 2.2. For any graph $G, O I R(G) \leq \gamma_{g r}^{Z}(G)$.

\section{Grundy domination number}

Brešar et al. [11] showed that the decision problem corresponding to the Grundy domination number is NP-complete, even for chordal graphs, while $\gamma_{g r}(G)$ can be computed in polynomial time for trees, cographs and split graphs. They determined several bounds on the Grundy domination number. In this section we begin with bounds on the Grundy domination number and then turn our attention to the Grundy domination number of product graphs.

\subsection{Bounds on $\gamma_{g r}(G)$}

In the paper introducing Grundy dominating sequences, Brešar et al. [11] observed the following.

Proposition 3.1 (Brešar et al. [11]). For a graph $G$ of order $n$ and minimum degree $\delta(G), \gamma_{g r}(G) \leq n-\delta(G)$.

Proof. Let $S=\left(v_{1}, v_{2}, \ldots, v_{k}\right)$ be a $\gamma_{g r}$-sequence of $G$. Let $u \in N\left[v_{k}\right]$ be a vertex that is not dominated by $\widehat{S}_{k-1}$, that is, $N[u] \cap \widehat{S}_{k-1}=\emptyset$. Therefore, $\left|\widehat{S}_{k-1}\right|=k-1 \leq|V|-|N[u]|=n-(d(u)+1)$. Thus, $\gamma_{g r}(G)=k \leq n-\delta(G)$.

A vertex $v$ in a set $S \subseteq V$ is called irredundant (with respect to $S$ ) if $N[v] \backslash N[S \backslash\{v\}] \neq \emptyset$, that is, $\operatorname{pn}[v, S] \neq \emptyset$. The upper irredundance number $I R(G)$ equals the maximum cardinality of an irredundant set $S$ in $G$. Thus, if a set $\left\{v_{1}, v_{2}, \ldots, v_{k}\right\}$ is a maximum cardinality irredundant set, then the sequence $\left(v_{1}, v_{2}, \ldots, v_{k}\right)$ is automatically a closed neighborhood sequence, and the following result is apparent.

Proposition 3.2. For any graph $G, I R(G) \leq \gamma_{g r}(G)$.

It is important to note that the value of $\gamma_{g r}(G)$ can be arbitrarily larger than $I R(G)$, since for the path $P_{n}, \operatorname{IR}\left(P_{n}\right)=$ $\lceil n / 2\rceil<\gamma_{g r}\left(P_{n}\right)=n-1$.

Proposition 3.3. For any graph $G$, $\operatorname{diam}(\mathrm{G}) \leq \gamma_{\mathrm{gr}}(\mathrm{G})$.

Proof. Let $v_{1}, v_{2}, \ldots, v_{k}, v_{k+1}$ be a shortest path between vertices $v_{1}$ and $v_{k+1}$ in $G$, where $k=\operatorname{diam}(\mathrm{G})$. It follows that $\left(v_{1}, v_{2}, \ldots, v_{k}\right)$ is a closed neighborhood sequence. Therefore, $k=\operatorname{diam}(\mathrm{G}) \leq \gamma_{\mathrm{gr}}(\mathrm{G})$.

Note that $I R(G)$ and $\operatorname{diam}(\mathrm{G})$ are not comparable, as can be seen by considering the $\operatorname{star} G=K_{1, n-1}$, for which $\operatorname{diam}(\mathrm{G})=$ $2<\mathrm{IR}(\mathrm{G})=\mathrm{n}-1$, and the path $P_{n}$, for which $\operatorname{IR}\left(P_{n}\right)=\lceil n / 2\rceil<\operatorname{diam}\left(\mathrm{P}_{\mathrm{n}}\right)=\mathrm{n}-1$.

Brešar et al. [11] showed that every graph $G$ has a maximal dominating sequence of length $k$, for every $k, \gamma(G) \leq k \leq$ $\gamma_{g r}(G)$. They also characterized the graphs $G$ for which $\gamma(G)=\gamma_{g r}(G)=k$, for $k \leq 3$.

An edge-clique cover of $G$ is a set $\mathcal{C}=\left\{C_{1}, C_{2}, \ldots, C_{k}\right\}$ of cliques of $G$ such that every edge $u v \in E$ is contained in a clique of $\mathcal{C}$. The edge-clique cover number $\theta_{e}(G)$ equals the smallest integer $k$ such that $G$ has an edge-clique cover of order $k$.

Proposition 3.4 (Brešar et al. [8]). For any isolate-free graph $G, \gamma_{g r}(G) \leq \theta_{e}(G)$. 
Proof. Let $\mathcal{C}=\left\{C_{1}, C_{2}, \ldots, C_{r}\right\}$ be a minimum order, edge-clique cover of an isolate-free graph $G$, that is, $r=\theta_{e}(G)$, and let $S=\left(v_{1}, v_{2}, \ldots, v_{k}\right)$ be a $\gamma_{g r}$-sequence of $G$. We claim that for every vertex $v_{i}$ in $S$, there exists a new clique $C_{j} \in \mathcal{C}$, which is not dominated by $\widehat{S}_{i-1}$, but is dominated by $\widehat{S}_{i}$. Let $x$ be a private neighbor of $v_{i}$ with respect to $\widehat{S}_{i-1}$, where possibly $x=v_{i}$. If $x \neq v_{i}$, consider the edge $e=v_{i} x$, and if $x=v_{i}$, consider the edge $e=v_{i} y$, where $y$ is any neighbor of $v_{i}$ (such a neighbor must exist since $G$ is isolate-free). In either case the edge $e$ lies in a clique, say $C_{j}$, that is not yet completely dominated, since either $v_{i}$ or $x$, or both, are not dominated by $\widehat{S}_{i-1}$. It follows that after vertex $v_{i}$ is selected, every vertex of clique $C_{j}$ is dominated. Therefore, $\mathcal{C}$ must contain at least $k=\gamma_{g r}(G)$ cliques.

Next we define a new vertex sequence that yields an upper bound on the Grundy domination number.

Given a vertex set $S \subseteq V$ in a graph $G$, a vertex $v \in S$ is called an enclave if $N[v] \subseteq S$, which is to say that vertex $v$ has no neighbors in $\bar{S}$. A set $S$ is called enclaveless if it contains no enclaves. A sequence $S=\left(v_{1}, v_{2}, \ldots, v_{k}\right)$ is called an enclaveless sequence if for every vertex $v_{i}$, where $i \in\{2,3, \ldots, k\}, v_{i}$ is not an enclave in the set $\widehat{S}_{i}$. Let the length of a longest enclaveless sequence be called the enclaveless sequence number, denoted by $\gamma_{e n}(G)$. Our next result shows that the enclaveless sequence number is an upper bound on the Grundy domination number.

Proposition 3.5. For any connected graph $G, \gamma_{g r}(G) \leq \gamma_{e n}(G)$.

Proof. Let $S=\left(v_{1}, v_{2}, \ldots, v_{k}\right)$ be a $\gamma_{g r}$-sequence of $G$, that is, $k=\gamma_{g r}(G)$. By definition, every vertex $v_{i} \in \widehat{S}$ has a vertex $x$ in its closed neighborhood $N\left[v_{i}\right]$ that is not dominated by any vertex in $\widehat{S}_{i-1}$. If $x=v_{i}$, then $v_{i}$ is not adjacent to any vertex in $\widehat{S}_{i-1}$. And since $G$ is connected, $v_{i}$ must have a neighbor $V \backslash \widehat{S}_{i-1}$. It follows, therefore, that $v_{i}$ is not an enclave in $\widehat{S}_{i}$. If $x \neq v_{i}$, then $x \notin \widehat{S}_{i-1}$, and so $v_{i}$ is not an enclave in $\widehat{S}_{i}$. Therefore, $S$ is an enclaveless sequence, and so $\gamma_{g r}(G) \leq \gamma_{e n}(G)$.

It is worth noting that the inequality in Proposition 3.5 is strict for nontrivial complete graphs $K_{n}$, since $\gamma_{g r}\left(K_{n}\right)=1<$ $\gamma_{e n}\left(K_{n}\right)=n-1$.

Since being developed by Cockayne, Hedetniemi, and Miller [21] in 1978, the domination chain, relating domination, independence and irredundance numbers, has been the focus of several hundred research papers. The upper portion of the chain states, for any graph $G$, that $\alpha(G) \leq \Gamma(G) \leq I R(G)$. As we observed in Proposition 3.2, for any graph $G$, $I R(G) \leq \gamma_{g r}(G)$. Thus, we have the following corollary to Proposition 3.5, which extends the domination chain.

\section{Corollary 3.1. For any connected graph $G$,}

$$
\alpha(G) \leq \Gamma(G) \leq I R(G) \leq \gamma_{g r}(G) \leq \gamma_{e n}(G) .
$$

Another parameter comes into focus from a concept that was introduced in the 1997 papers by Cockayne, Grobler, Hedetniemi, and McRae [19] and Cockayne, Hattingh, Hedetniemi, Hedetniemi, and McRae [20].

A set $S$ is called external redundant if for every vertex $v \in \bar{S}$, either (i) $v$ does not have a private neighbor with respect to $S \cup\{v\}$ or (ii) $v$ has a private neighbor, but there exists some vertex $u \in S$ having a private neighbor with respect to $S$ but no private neighbor with respect to $S \cup\{v\}$. Let $E R(G)$ equal the maximum cardinality of a minimal external redundant set in $G$. Notice that an external redundant set need not be irredundant, but, by definition, every maximal irredundant set is external redundant.

In [19] and [20] the following inequalities are established for any graph $G$ :

$$
\alpha(G) \leq \Gamma(G) \leq I R(G) \leq E R(G) .
$$

This raises the question of the relationship, if any, between $\gamma_{g r}(G)$ and $E R(G)$.

\subsection{Grundy domination number in grids, cylinders and tori}

In 2016 Brešar, Butjás, Gologranc, Klavžar, Košmrlj, Pakkós, Tuza, and Vizer [8] studied the Grundy domination number in the four standard graph products: the Cartesian, lexicographic, direct and strong product. For each of these graph products they presented lower bounds for the Grundy domination number, which are exact for the lexicographic product and they conjectured are exact for the strong product. In most cases they completely determined the Grundy domination numbers for products of paths (grid graphs), paths and cycles (cylinders), and cycles (tori). In this subsection we will review only their results for Cartesian products of paths and cycles.

We first give some additional terminology. The Cartesian product $G \square H$ of two graphs $G$ and $H$ is the graph $G \square H=$ $(V(G) \times V(H), E(G \square H))$, whose vertex set is the set of all ordered pairs $(u, v)$, where $u \in V(G)$ and $v \in V(H)$. Two vertices $\left(u_{1}, v_{1}\right)$ and $\left(u_{2}, v_{2}\right)$ are adjacent in $G \square H$, if and only if either $u_{1}$ is adjacent to $u_{2}$ in $G$ and $v_{1}=v_{2}$, or $u_{1}=u_{2}$ and $v_{1}$ is adjacent to $v_{2}$ in $H$. 
A grid graph is a Cartesian product of two paths, denoted by $G_{m, n}=P_{m} \square P_{n}$; a cylinder is a Cartesian product $P_{m} \square C_{n}$ of a path and a cycle or $C_{m} \square P_{n}$ of a cycle and a path; and a torus is a Cartesian product $C_{m} \square C_{n}$ of two cycles.

A lower bound on the Grundy domination number of the Cartesian product of general graphs is given in [8].

Theorem 3.1 (Brešar et al. [8]). For any two graphs $G$ and $H$,

$$
\gamma_{g r}(G \square H) \geq \max \left\{\gamma_{g r}(G)|V(H)|, \gamma_{g r}(H)|V(G)|\right\} .
$$

Theorem 3.2 (Brešar et al. [8]). For any paths $P_{m}$ and $P_{n}$, and any cycles $C_{m}$ and $C_{n}$,

(i) $\gamma_{g r}\left(P_{m} \square P_{n}\right)=m(n-1)$, for $2 \leq m \leq n$;

(ii) $\gamma_{g r}\left(P_{m} \square C_{n}\right)=\max \{n(m-1), m(n-2)\}$, for $2 \leq m$ and $3 \leq n$;

(iii) $\gamma_{g r}\left(C_{m} \square C_{n}\right)=m(n-2)$, for $3 \leq m \leq n$ and $(m, n) \neq(2 t+1,2 t+1)$ for some $1 \leq t$;

(iv) $\gamma_{g r}\left(C_{m} \square C_{n}\right)=m(m-2)+1$, for $m$ odd.

For (i) grid graphs $P_{m} \square P_{n}$, having $n$ columns of height $m$, for $m \leq n$, and vertices $V\left(P_{m}\right)=\left\{u_{1}, u_{2}, \ldots, u_{m}\right\}$ and $V\left(P_{n}\right)=\left\{v_{1}, v_{2}, \ldots, v_{n}\right\}$, a maximum length dominating sequence $S$ is obtained by listing the vertices, column-by-column, that is, listing all vertices in the first column, then all vertices in the second column, and continuing until listing all vertices in the $(n-1)^{s t}$ column. Specifically,

$$
S=\left(\left(u_{1}, v_{1}\right), \ldots,\left(u_{m}, v_{1}\right),\left(u_{1}, v_{2}\right), \ldots,\left(u_{m}, v_{2}\right), \ldots,\left(u_{1}, v_{n-1}\right), \ldots,\left(u_{m}, v_{n-1}\right)\right)
$$

is a $\gamma_{g r}$-sequence of $P_{m} \square P_{n}$.

For a torus (iii), the same listing of vertices is optimal, except that only vertices in the first $n-2$ columns are listed, since the vertices in the first column dominate all vertices in the $n^{\text {th }}$ column.

\section{Grundy total domination number}

Brešar, Henning, and Rall [12] proved an upper bound for the Grundy total domination number in terms of the order and minimum degree of a graph $G$, and a lower bound in terms of the order and maximum degree.

Theorem 4.1 (Brešar et al. [12]). For a graph $G$ with order n, minimum degree $\delta=\delta(G)$, and maximum degree $\Delta=\Delta(G)$,

$$
\frac{n}{\Delta} \leq \gamma_{g r}^{t}(G) \leq n-\delta+1
$$

The authors showed that $\gamma_{g r}^{t}(G)=\frac{n}{\Delta}$ if and only if $G$ is the complete bipartite graph $K_{\Delta, \Delta}$, and they left characterizing the graphs obtaining the upper bound of Theorem 4.1 as an open problem.

The Grundy total domination numbers of paths $P_{n}$ and cycles $C_{n}$ were also determined in [12].

Proposition 4.1 (Brešar et al. [12]). For paths $P_{n}$,

$$
\gamma_{g r}^{t}\left(P_{n}\right)=\left\{\begin{array}{cl}
n & \text { if } n \geq 2 \text { even } \\
n-1 & \text { if } n \geq 3 \text { odd }
\end{array}\right.
$$

Proposition 4.2 (Brešar et al. [12]). For cycles $C_{n}$,

$$
\gamma_{g r}^{t}\left(C_{n}\right)= \begin{cases}n-2 & \text { if } n \geq 4 \text { even } \\ n-1 & \text { if } n \geq 3 \text { odd }\end{cases}
$$

For example, to see that a path $P_{n}=v_{1}, v_{2}, \ldots, v_{n}$ of even order $n$ has $\gamma_{g r}^{t}\left(P_{n}\right)=n$, consider the total dominating sequence $\left(v_{1}, v_{3}, \ldots, v_{n-1}, v_{n}, v_{n-2}, \ldots, v_{2}\right)$.

As observed in [12], $2 \leq \gamma_{t}(G) \leq \Gamma_{t}(G) \leq \gamma_{g r}^{t}(G) \leq n$.

Brešar et al. characterized the graphs $G$ having $\gamma_{g r}^{t}(G)=2$ as follows.

Theorem 4.2 (Brešar et al. [12]). A graph $G$ has $\gamma_{g r}^{t}(G)=2$ if and only if $G$ is a complete multipartite graph.

Proof. It is straightforward to show that a complete multipartite graph has $\gamma_{g r}^{t}(G)=2$.

For the converse, assume that $G$ is a graph with $\gamma_{g r}^{t}(G)=2$. We first note that for any pair of nonadjacent vertices $x$ and $y, N(x)=N(y)$, for otherwise $(x, y)$ or $(y, x)$ is an open neighborhood sequence that is not a total dominating sequence, implying that $\gamma_{g r}^{t}(G)>2$, a contradiction. Similarly, for any two adjacent vertices $x$ and $y$ of $G$, the sequence $(x, y)$ is an open neighborhood sequence, so it must be a total dominating sequence.

Thus, if $I$ is a maximal independent set in $G$, then every vertex in $I$ has the same open neighborhood. We claim that $N(x)=V \backslash I$ for all $x \in I$. Let $x \in I$ and suppose that there is a vertex $y \in V \backslash I$ that is not adjacent to $x$. Then $y$ must be 
adjacent to some vertex $w \in I$ for otherwise $I$ is not a maximal independent set. But then $x$ and $w$ are nonadjacent vertices with $N(x) \neq N(w)$, a contradiction. Therefore, $N(x)=V \backslash I$ for all $x \in I$. Since $I$ was an arbitrarily chosen maximal independent set, it follows that $G$ can be partitioned into maximal independent sets, each of which is adjacent to all other vertices not in that set. This implies that $G$ is a complete multipartite graph.

It is shown in [14] that there are no graphs $G$ with Grundy total domination number equal to 3.

Theorem 4.3 (Brešar et al. [14]). There exists no graph $G$ such that $\gamma_{g r}^{t}(G)=3$.

On the other hand, they showed that every positive integer except 1 and 3 can be realized as a Grundy total domination number. Let $\mathbb{N}$ denote the set of positive integers.

Theorem 4.4 (Brešar et al. [14]). For every $k \in \mathbb{N} \backslash\{1,3\}$, there exists a graph $G_{k}$ such that $\gamma_{g r}^{t}(G)=k$.

Proof. Let $G_{k}$ be the prism $K_{k} \square K_{2}$, and denote the vertices of one $k$-clique by $a_{1}, a_{2}, \ldots, a_{k}$ and of the other by $b_{1}, b_{2}, \ldots, b_{k}$, where $a_{i} b_{i} \in E\left(G_{k}\right)$. Note that $G_{2}=K_{2} \square K_{2}$ is the cycle $C_{4}$, and so $\gamma_{g r}^{t}\left(G_{2}\right)=2$.

Next consider $G_{k}$ for $k \geq 4$. The sequence $\left(a_{1}, a_{2}, \ldots, a_{k}\right)$ is a total dominating sequence of $G_{k}$. Thus, $\gamma_{g r}^{t}\left(G_{k}\right) \geq k$. Since every subset of $k$ vertices is a total dominating set of $G_{k}$, it follows that $\gamma_{g r}^{t}\left(G_{k}\right) \leq k$, and so $\gamma_{g r}^{t}\left(G_{k}\right)=k$.

Brešar et al. [14] also presented a family of graphs $G_{k}$ having the minimum possible order and size among all graphs with Grundy total domination number equal to $k$. If $k$ is even, then $G_{k}$ is the path $P_{k}$. Let $k \geq 5$ be odd. For $n=5$, the graph $G_{5}$ of order 6 obtained from two disjoint triangles by adding an edge between one vertex in each triangle has the minimum number of vertices and edges. For $k=5+2 j$, let $G_{k}$ be the graph obtained from $G_{5}$ by attaching a path $P_{2 j}$ to a vertex of $G_{5}$ with degree 2 . The graph $G_{9}$ is shown in Figure 1 for example.

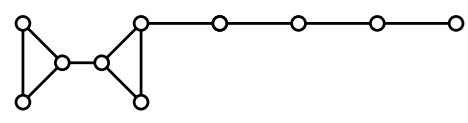

Figure 1: The graph $G_{9}$.

Let $M$ be a matching of $G$ and let $V(M)$ denote the set of vertices incident to the edges of $M$. If $V(M)=V(G)$, then $M$ is called a perfect matching. A vertex of $V(M)$ is called strong if it has degree 1 in the induced subgraph $G[V(M)]$. A matching $M$ in $G$ is a strong matching (also called an induced matching in the literature) if every vertex in $V(M)$ is strong. The number of edges in a maximum matching of $G$ is the matching number, denoted by $\alpha^{\prime}(G)$, and the number of edges in a maximum induced matching of $G$ is the strong matching number, denoted by $\alpha_{s}^{\prime}(G)$. A matching $M$ is called semistrong if every edge in $M$ is incident to a strong vertex. The number of edges in a maximum semistrong matching of $G$ is the semistrong matching number $\alpha_{s s}^{\prime}(G)$. Recall that the vertex cover number $\beta(G)$ is the minimum number of vertices of $G$ necessary to cover all the edges of $G$.

A lower bound on the Grundy total domination number based on the semistrong matching number is given in [12], while an upper bound in terms of the vertex cover number $\beta(G)$ is given in [14]. We state these bounds:

Theorem 4.5. For any graph $G$ without isolated vertices, $2 \alpha_{s s}^{\prime}(G) \leq \gamma_{g r}^{t}(G) \leq 2 \beta(G)$.

Corollary 4.1. For every bipartite graph $G$ without isolated vertices,

$$
2 \alpha_{s s}^{\prime}(G) \leq \gamma_{g r}^{t}(G) \leq 2 \alpha^{\prime}(G) .
$$

Brešar et al. [12] characterized the trees having Grundy total domination number equal to their order.

Theorem 4.6 (Brešar et al. [12]). If $T$ is a tree of order $n$, then $\gamma_{g r}^{t}(T)=n$ if and only if $T$ has a perfect matching.

To present the next result, we define a family of trees. A vertex that is adjacent to a leaf is called a support vertex, and a strong support vertex is adjacent to two or more leaves. Let $\mathcal{T}$ be the family of trees $T$ that contain a path $P_{2}$ and are closed under the operation $\mathcal{O}_{1}$, which extends a tree $T^{\prime}$ by adding a path $v_{1}, v_{2}, v_{3}$ and the edge $v v_{1}$ to a support vertex $v$ in the tree $T^{\prime}$. Note that every tree $T \in \mathcal{T}$ has order $n$ congruent to 2 modulo 3 . The two smallest trees in the family $\mathcal{T}$ are the path $P_{2}$ and the path $P_{5}$. Note also that the path $P_{5}$ is obtained from the path $P_{2}$ by applying operation $\mathcal{O}_{1}$ once (for the path $P_{2}$, we consider one vertex to be a support vertex). See Figure 2 for the tree obtained by applying operation $\mathcal{O}_{1}$ to the path $P_{5}$.

Theorem 4.7 (Brešar et al. [12]). If $T$ is a nontrivial tree of order $n$ with no strong support vertex, then $\gamma_{g r}^{t}(T) \geq \frac{2}{3}(n+1)$, with equality if and only if $T \in \mathcal{T}$. 


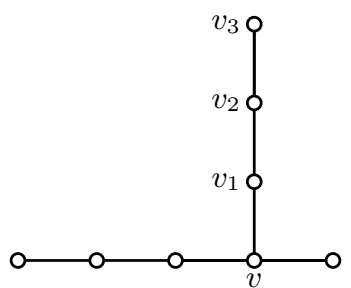

Figure 2: $\mathcal{O}_{1}$ applied to $P_{5}$.

A lower bound on the Grundy total domination number of connected $r$-regular graphs different from the complete bipartite graph $K_{r, r}$ is given in [12].

Theorem 4.8 (Brešar et al. [12]). For $r \geq 3$, if $G$ is a connected $r$-regular graph of order $n$ different from $K_{r, r}$, then

$$
\gamma_{g r}^{t}(G) \geq \begin{cases}\frac{n+\left\lceil\frac{r}{2}\right\rceil-2}{r-1} & \text { if } G \text { is not bipartite, } \\ \frac{n+2\left\lceil\frac{r}{2}\right\rceil-4}{r-1} & \text { if } G \text { is bipartite. }\end{cases}
$$

Corollary 4.2. If $G$ is a connected cubic graph of order $n$ different from $K_{3,3}$, then $\gamma_{g r}^{t}(G) \geq \frac{1}{2} n$.

Brešar et al. [12] proved that the decision problem associated with the Grundy total domination number is NP-complete, even when restricted to bipartite graphs. Brešar et al. [14] showed that this is also true when restricted to split graphs. On the other hand, the authors of [14] gave a linear time algorithm for determining the Grundy total domination number of any isolate-free forest and a similar algorithm for bipartite distance-hereditary graphs. They also presented a framework for obtaining polynomial algorithms to solve this problem in other classes of graphs.

In conclusion, we note that Brešar et al. [12] showed that twice the Grundy domination number is an upper bound on the Grundy total domination number.

\section{Towards a theory of vertex sequences in graphs}

Motivated by the neighborhood (dominating) vertex sequences in the previous sections, let us consider vertex sequences in a more general setting. In the process, we will propose several new types of vertex sequences for further study, along with some preliminary results for them.

The vertex sequences $S=\left(v_{1}, v_{2}, \ldots, v_{k}\right)$ presented so far are all defined in terms of a requirement placed on the next vertex $v_{i}$ in the sequence with regard to either (i) the set $\widehat{S}_{i-1}$ of previous vertices in the sequence, or (ii) the set of vertices not in $\widehat{S}_{i-1}$.

The definitions of a walk, trail, path and cycle are well known in graph theory, and all of these are examples of vertex sequences in which a very simple requirement is placed on the next vertex $v_{i}$ in the sequence, namely that the next vertex must be adjacent to the immediately preceding vertex.

A walk $w$ in a graph $G$ from a vertex $u$ to a vertex $v$ is a finite, alternating sequence of vertices and edges, $W=$ $u, e_{1}, u_{1}, e_{2}, u_{2}, \ldots, e_{k}, v$, starting with the vertex $u$ and ending with the vertex $v$, in which each edge $e_{i}=u_{i-1} u_{i}$ of the sequence joins the vertex that precedes it in the sequence to the vertex that follows it in the sequence.

Equivalently, we can say that a walk is a vertex sequence $W=\left(v_{1}, v_{2}, \ldots, v_{k}\right)$, in which every vertex $v_{i}$ must be adjacent to the vertex $v_{i-1}$ preceding it in the sequence. Notice that in the definition of a walk, both vertices and edges may be repeated.

A trail is a walk having no repeated edges, or equivalently, a vertex sequence in which every vertex $v_{i}$ is adjacent to the vertex $v_{i-1}$ preceding it in the sequence, and $v_{i}$ does not appear consecutively with $v_{i-1}$ anywhere else in the sequence.

A path is a walk having no repeated vertices, that is, a vertex sequence in which every vertex $v_{i}$ is adjacent to the vertex $v_{i-1}$ preceding it in the sequence, and in which no vertex appears more than once.

A cycle is a walk $W=\left(v_{1}, v_{2}, \ldots, v_{k}\right)$ in which $v_{1}=v_{k}$ and no other vertices are repeated.

Vertex sequences appear in many places in graph theory, such as the following. For any graph $G$ :

1. the Hamiltonian completion number equals the minimum length of a walk containing all vertices of $G$.

2. an Eulerian walk is a walk of minimum length containing all edges of $G$.

3. the trail number equals maximum length of a trail in $G$. 
4. the detour number equals the maximum length of a path in $G$.

5. the diameter equals maximum length of an induced path in $G$.

6. the circumference equals the maximum length of a cycle in $G$.

7. the induced circumference equals the maximum length of an induced cycle in $G$.

8. the girth equals the minimum length of a cycle in $G$.

Motivated by these well known vertex sequences and the neighborhood (dominating) vertex sequences, we propose the following types of vertex sequences for further study.

\subsection{Double dominating sequences}

Recall that a vertex $v$ dominates all vertices in its closed neighborhood $N[v]$. A vertex set $D$ is a double dominating set of $G$ if every vertex in $V$ is dominated by at least two vertices in $D$, that is, $|N[v] \cap D| \geq 2$ for every $v \in V$. Note that double domination is only defined for graphs having no isolated vertices.

Let $S=\left(v_{1}, v_{2}, \ldots, v_{k}\right)$ be a sequence of vertices in a graph $G$ without isolated vertices such that every vertex $v_{i} \in S$ dominates at least one vertex $x$ in $V \backslash \widehat{S}_{i-1}$ that is dominated at most once by vertices in $\widehat{S}_{i-1}$. This means that $\left|N[x] \cap \widehat{S}_{i-1}\right| \leq$ 1. Once again, it is possible that $x=v_{i}$ if $\left|N\left(v_{i}\right) \cap \widehat{S}_{i-1}\right| \leq 1$. Such a sequence $S$ of maximal length is called a double dominating sequence since $\widehat{S}$ is a double dominating set of $G$. The maximum length of a double dominating sequence is called the Grundy double domination number, denoted by $\gamma_{2 g r}(G)$. A double dominating sequence of length $\gamma_{2 g r}(G)$ is called a $\gamma_{2 g r}$-sequence.

In order to illustrate this straightforward generalization of dominating sequences, consider the cycle $C_{6}$, with vertices labeled in order $v_{1}, v_{2}, v_{3}, v_{4}, v_{5}, v_{6}$. The vertex sequence $\left(v_{1}, v_{2}, v_{3}, v_{4}\right)$ can be seen to be a maximum length dominating sequence and $\gamma_{g r}\left(C_{6}\right)=4$, while the sequence $\left(v_{1}, v_{2}, v_{3}, v_{4}, v_{5}\right)$ is a $\gamma_{2 g r}$-sequence and $\gamma_{2 g r}\left(C_{6}\right)=5$. Note that in general, for any graph $G, \gamma_{g r}(G) \leq \gamma_{2 g r}(G)$.

We observe that for any nontrivial path $P_{n}$,

$$
\gamma_{g r}\left(P_{n}\right)=n-1<\gamma_{2 g r}\left(P_{n}\right)=n,
$$

and for any cycle $C_{n}$,

$$
\gamma_{g r}\left(C_{n}\right)=n-2<\gamma_{2 g r}\left(C_{n}\right)=n-1 .
$$

Let $G$ be a graph with order $n$ and minimum degree $\delta(G) \geq 2$. Since for any sequence $S$ of $n-1$ vertices of $G$ that omits a vertex, say $x, \widehat{S}$ double dominates $x$, we make the following observation.

Observation 5.1. For any graph $G$ with order $n$ and $\delta(G) \geq 2, \gamma_{2 g r}(G) \leq n-1$.

For an example of graphs having Grundy double domination number one less than their order, we consider grid graphs. Recall Theorem 3.2, by Brešar et al. [8]:

For any paths $P_{m}$ and $P_{n}, \gamma_{g r}\left(P_{m} \square P_{n}\right)=m(n-1)$, for $2 \leq m \leq n$. The corresponding result for double dominating sequences is the following.

Theorem 5.1. For any paths $P_{m}$ and $P_{n}, \gamma_{2 g r}\left(P_{m} \square P_{n}\right)=m n-1$, for $2 \leq m \leq n$.

Proof. For grid graphs $P_{m} \square P_{n}$, having $n$ columns of height $m$, for $2 \leq m \leq n$, and vertices $V\left(P_{m}\right)=\left\{u_{1}, u_{2}, \ldots, u_{m}\right\}$ and $V\left(P_{n}\right)=\left\{v_{1}, v_{2}, \ldots, v_{n}\right\}$, the sequence obtained by simply listing the vertices, column-by-column, that is, listing all vertices in the first column, then all vertices in the second column, and continuing until listing all vertices in the $n^{\text {th }}$ column except for the last vertex $\left(u_{m}, v_{n}\right)$ (which will have been dominated twice):

$$
S=\left(\left(u_{1}, v_{1}\right), \ldots,\left(u_{m}, v_{1}\right),\left(u_{1}, v_{2}\right), \ldots,\left(u_{m}, v_{2}\right), \ldots,\left(u_{1}, v_{n}\right), \ldots,\left(u_{m-1}, v_{n}\right)\right)
$$

is a double dominating sequence of length $m n-1$. Since $P_{m} \square P_{n}$ has order $m n$, it follows from Observation 5.1 that $\gamma_{2 g r}\left(P_{m} \square P_{n}\right)=m n-1$.

On the other hand, trees have Grundy double domination number equal to their order.

Theorem 5.2. For any tree $T$ of order $n \geq 2, \gamma_{2 g r}(T)=n$. 
Proof. Let $T$ be a tree with vertices $V=\left\{v_{1}, v_{2}, \ldots, v_{n}\right\}$, listed in breadth-first order, as follows. Assume that $T$ is rooted at vertex $v_{1}$. Let $D_{i}=\left\{v_{j} \mid d\left(v_{j}, v_{1}\right)=i\right\}$ be the set of vertices at distance $i$ from the root $v_{1}$. Let the maximum distance from $v_{1}$ to a vertex in $T$ be $k$, and consider the sequence of sets $D_{1}, D_{2}, \ldots, D_{k}$. Form a sequence of vertices in $V$ by listing $v_{1}$ first, followed by all vertices, in any order, in set $D_{1}$ (the children of vertex $v_{1}$ ), then by all the vertices in $D_{2}$, etc., until all vertices have been listed, and we have the sequence $S=\left(v_{1}, v_{2}, \ldots, v_{n}\right)$. Consider any vertex $v_{i}$ for $i \geq 2$ in this sequence. It has been dominated exactly once, by its parent in $\widehat{S}_{i-1}$. Thus, it qualifies to be listed next after the sequence $v_{1}, v_{2}, \ldots, v_{i-1}$ according to the sequence definition. Hence, $S$ is a double dominating sequence, and so $\gamma_{2 g r}(T)=n$.

Let $G-x$ denote the graph formed by removing the vertex $x$ and its incident edges from a graph $G$.

Proposition 5.1. If $G$ is a connected graph of order $n \geq 3$ with a vertex $x$ of degree 1 , then $\gamma_{2 g r}(G) \geq \gamma_{2 g r}(G-x)+1$.

Proof. Let $G$ be a connected graph of order $n \geq 3$ and let $x$ be a vertex of $G$ with degree 1 . Note that $G-x$ is connected and has minimum degree at least 1 . Let $S_{x}$ be a $\gamma_{2 g r}$-sequence of $G-x$. Now $\widehat{S}_{x}$ dominates $x$ at most once, so adding $x$ to the end of $S_{x}$ is allowed when building a double dominating sequence of $G$. Thus, $\gamma_{2 g r}(G) \geq \gamma_{2 g r}(G-x)+1$.

We note that double dominating sequences can be generalized to $k$-tuple dominating sequences by requiring that for each vertex $v_{i}$ in the sequence $S=\left(v_{1}, v_{2}, \ldots, v_{s}\right)$, there exists a vertex $x \in N\left[v_{i}\right]$ such that $\left|N[x] \cap \widehat{S}_{i-1}\right| \leq k-1$.

\subsection{Connected dominating sequences}

A dominating set $D$ is a connected dominating set of a graph $G$ if the induced subgraph $G[D]$ is connected. The connected domination number $\gamma_{c}(G)$ equals the minimum cardinality of a connected dominating set of $G$.

Let $S=\left(v_{1}, v_{2}, \ldots, v_{k}\right)$ be a sequence of vertices in a connected graph $G$ such that for $2 \leq i \leq k$, every vertex $v_{i}$ in $S$ is adjacent to at least one vertex in $\widehat{S}_{i-1}$ and dominates at least one previously undominated vertex. In other words, $S$ is a closed neighborhood sequence with the added restriction that every $v_{i}$, for $2 \leq i \leq k$, has a neighbor in $\widehat{S}_{i-1}$. Such a sequence $S$ of maximal length is called a connected dominating sequence since $\widehat{S}$ is a connected dominating set of $G$. The maximum length of a connected dominating sequence is called the Grundy connected domination number and is denoted by $\gamma_{g r}^{c}(G)$.

We first show that the minimum length of a connected dominating sequence of a graph $G$ equals the connected domination number of $G$.

Theorem 5.3. For any connected graph $G$, the minimum length of a connected dominating sequence of $G$ equals its connected domination number $\gamma_{c}(G)$.

Proof. Let $S=\left(v_{1}, v_{2}, \ldots, v_{k}\right)$ be a connected dominating sequence of minimum length. Since by definition $\widehat{S}$ is a connected dominating set of $G$, we have $\gamma_{c}(G) \leq|\widehat{S}|=k$.

Recall that by definition a connected dominating sequence has maximal length. Hence, to show that $k=\gamma_{c}(G)$, it suffices to show that there exists a connected dominating sequence of $G$ having length $\gamma_{c}(G)$. Let $D$ be a minimum connected dominating set of $G$, that is, $|D|=\gamma_{c}(G)$. Then $G[D]$ is connected. Consider a spanning tree $T$ of $G[D]$.

Assume that $T$ is rooted at vertex $v_{1}$. Let $D_{i}=\left\{v_{j} \mid d_{T}\left(v_{j}, v_{1}\right)=i\right\}$ be the set of vertices at distance $i$ in $T$ from the root $v_{1}$. Let the maximum distance from $v_{1}$ to a vertex in $T$ be $k$, and consider the sequence of sets $D_{1}, D_{2}, \ldots, D_{k}$. Form a sequence of vertices in $V$ by listing $v_{1}$ first, followed by all vertices, in any order, in set $D_{1}$ (the children of vertex $v_{1}$ ), then by all vertices in $D_{2}$, etc., until all vertices have been listed, and we have the sequence $S=\left(v_{1}, v_{2}, \ldots, v_{\gamma_{c}}\right)$. For $i \geq 2$, the vertex $v_{i}$ has a neighbor, namely its parent in $T$, in the set $\widehat{S}_{i-1}$. If there exists a vertex $x \in N\left[v_{i}\right]$ that is not dominated by $\widehat{S}_{i-1}$ in $G$, then $v_{i}$ qualifies to be listed next after the sequence $v_{1}, v_{2}, \ldots, v_{i-1}$ according to the sequence definition. If this holds for all $v_{i}$, then we have the desired result.

Suppose, to the contrary, that there exists a vertex $v_{i}$ such that $N\left[v_{i}\right]$ is dominated by $\widehat{S}_{i-1}$ in $G$. Then either $v_{i}$ has no children in $T$, that is, $v_{i}$ is a leaf in $T$, or every child of $v_{i}$ has a neighbor in $\widehat{S}_{i-1}$ in $G[D]$. In either case, the induced subgraph $G\left[D \backslash\left\{v_{i}\right\}\right]$ is connected, implying that $D \backslash\left\{v_{i}\right\}$ is a connected dominating set of $G$ with cardinality less than $\gamma_{c}(G)$, a contradiction. Hence, $S$ is a connected dominating sequence of length $\gamma_{c}(G)$, as desired.

Using an identical argument to the proof of Proposition 3.1, we can show the following.

Proposition 5.2. For any connected graph $G$ of order $n$ and minimum degree $\delta(G), \gamma_{g r}^{c}(G) \leq n-\delta(G)$.

Corollary 5.1. For any connected graph $G$ of order $n \geq 2, \gamma_{g r}^{c}(G) \leq n-1$. 
Similarly, an identical proof to the one given for Proposition 3.3 shows that the diameter of a connected graph $G$ is a lower bound on the Grundy connected domination number.

Proposition 5.3. For any nontrivial connected graph $G$,

$$
\operatorname{diam}(\mathrm{G}) \leq \gamma_{\mathrm{gr}}^{\mathrm{c}}(\mathrm{G})
$$

As a corollary to Propositions 5.2 and 5.3, we have the following.

Corollary 5.2. For paths $P_{n}$ with order $n \geq 2, \gamma_{g r}^{c}\left(P_{n}\right)=n-1$.

\subsection{Vertex cover sequences}

A set $X$ of vertices of $G$ is a vertex cover if every edge in $G$ is incident to at least one vertex of $X$. The vertex cover number $\beta(G)$ equals the minimum cardinality of a vertex cover of $G$.

Let $S=\left(v_{1}, v_{2}, \ldots, v_{k}\right)$ be a sequence of vertices in a graph $G$ such that for $2 \leq i \leq k$, every vertex $v_{i}$ in $S$ is incident to at least one uncovered edge $v_{i} w$, that is, such that $w \notin \widehat{S}_{i-1}$. Such a sequence $S$ of maximal length is called a vertex cover sequence since $\widehat{S}$ is a vertex cover of $G$. The maximum length of a vertex cover sequence is called the Grundy vertex cover number, denoted by $\beta_{g r}(G)$.

The following result is immediate.

Proposition 5.4. For any graph $G$, the minimum length of a vertex cover sequence equals the vertex cover number $\beta(G)$.

Define $\beta^{+}(G)$ to equal the maximum cardinality of a minimal vertex cover. As an illustration of this, consider a path $P_{3}$ with vertices in order $u, v, w$. The set $S=\{v\}$ is a minimum cardinality vertex cover of $P_{3}$, that is, $\beta\left(P_{3}\right)=1$. However, the set $S^{\prime}=\{u, w\}$ is a minimal vertex cover of maximum cardinality, that is, $\beta^{+}\left(P_{3}\right)=2$.

Proposition 5.5. For any graph $G$ of size $m=|E|, \beta^{+}(G) \leq \beta_{g r}(G) \leq m$.

Proof. Let $S=\left\{v_{1}, v_{2}, \ldots, v_{k}\right\}$ be a minimal vertex cover of maximum cardinality, where $k=\beta^{+}(G)$. Since $S$ is a minimal vertex cover, every vertex in $S$ covers at least one edge that no other vertex in $S$ covers. Thus, the sequence $S^{\prime}=\left(v_{1}, v_{2}, \ldots, v_{k}\right)$ is a vertex cover sequence. Hence, $k=\beta^{+}(G) \leq \beta_{g r}(G)$. Since every vertex $v_{i}$ in a vertex cover sequence covers a previously uncovered edge, it is obvious that $\beta_{g r}(G) \leq m$.

A simple illustration of the Grundy vertex cover number is given by the complete graph, where $\beta_{g r}\left(K_{n}\right)=n-1$, while $m=\left(\begin{array}{l}n \\ 2\end{array}\right)$. Notice also that $\beta_{g r}\left(P_{n}\right)=n-1=m$.

We characterize the graphs attaining the upper bound of Proposition 5.5.

Theorem 5.4. A connected graph $G$ with size $m \geq 1$ has $\beta_{g r}(G)=m$ if and only if $G$ is a nontrivial tree.

Proof. Let $T$ be a nontrivial tree rooted at a vertex $v_{1}$, with vertices $V=\left\{v_{1}, v_{2}, \ldots, v_{n}\right\}$, listed in breadth-first order, as follows. Let $D_{i}=\left\{v_{j} \mid d\left(v_{j}, v_{1}\right)=i\right\}$ be the set of vertices at distance $i$ from the root $v_{1}$. Let the maximum distance from $v_{1}$ to a vertex in $T$ be $k$, and consider the sequence of sets $D_{k}, D_{k-1}, \ldots, D_{1}$. Form a sequence of vertices in $V$ by listing all vertices, in any order, in set $D_{k}$, followed by all vertices in $D_{k-1}$, etc., until all vertices in $D_{1}$ have been listed, and we have a sequence $S=\left(v_{1}, v_{2}, \ldots, v_{n-1}\right)$ of $n-1$ vertices of $T$, containing every vertex except the root vertex $v_{1}$. Since every vertex $v_{i}$ is listed before its parent, say $v_{j}$, in the sequence, the edge $v_{i} v_{j}$ is not covered by $\widehat{S}_{i-1}$, so $v_{i}$ qualifies to be listed next after the sequence $v_{i-1}$. Hence, $\beta_{g r}(T) \geq|S|=n-1$. But since there are only $n-1$ edges in a tree $T$ of order $n$, it follows that $\beta_{g r}(T)=n-1=m$.

Conversely, assume that $G$ is connected graph with $\beta_{g r}(G)=m \geq 1$. Suppose that $G$ is not a tree, that is, $G$ has at least one cycle subgraph, say $C_{j}$. Let $S$ be a vertex cover sequence of maximum length, that is, $\beta_{g r}(G)=|\widehat{S}|=m$. Since $\beta_{g r}(G)=m$, there is a 1-to-1 correspondence between the vertices of $\widehat{S}$ and the edges of $G$ such that each edge can be mapped to one of its endpoints in $\widehat{S}$. In particular, there is a 1-to-1 mapping between the $j$ edges on the cycle $C_{j}$ the $j$ vertices of $C_{j}$. Thus, every edge not on $C_{j}$ is mapped to a vertex in $\widehat{S}$ not on $C_{j}$. But no matter what order the vertices of $C_{j}$ appear in $S$, after $j-1$ vertices of $C_{j}$ are in the sequence, all the edges of $C_{j}$ are covered and the remaining cycle vertex does not qualify to be added to the sequence, a contradiction.

Corollary 5.3. For any cycle $C_{n}$ of order $n \geq 3, \beta_{g r}\left(C_{n}\right)=n-1$. 


\subsection{1-Adjacent vertex sequences}

A sequence $S=\left(v_{1}, v_{2}, \ldots, v_{k}\right)$ of vertices in a graph $G$ is called a 1-adjacent vertex sequence if for every $2 \leq i \leq k$, vertex $v_{i}$ in $S$ is adjacent to exactly one vertex in $\widehat{S}_{i-1}$. The maximum length of a 1-adjacent vertex sequence is called the Grundy 1-adjacent vertex number and is denoted by $\operatorname{gr}_{1 a}(\mathrm{G})$.

Proposition 5.6. If $S=\left(v_{1}, v_{2}, \ldots, v_{k}\right)$ is a maximal 1-adjacent vertex sequence in a graph $G$, then the induced subgraph $G\left[\widehat{S}_{k}\right]$ must be a tree and, in fact, is a maximal induced subtree of $G$.

Proof. Let $S=\left(v_{1}, v_{2}, \ldots, v_{k}\right)$ be a maximal 1-adjacent vertex sequence in a graph $G$. It follows from the definition of a 1-adjacent vertex sequence, and a simple induction on the length of the sequence, that the induced subgraph $G[\widehat{S}]$ is a connected subgraph. It remains to show that this subgraph must be a tree $T=G[\widehat{S}]$.

Assume not. Then let $C$ be a cycle in $G[\widehat{S}]$. Of all the vertices on this cycle $C$, let $v_{j}$ have the maximum index in $S$. Then $v_{j}$ must be adjacent to at least two vertices on $C$ and hence is adjacent to at least two vertices in $\widehat{S}_{k-1}$ having smaller indices. This is a contradiction to the rule of adding $v_{j}$ to the sequence $S$ if and only if it is adjacent to exactly one vertex having smaller index.

It follows that $T$ must be a maximal induced subtree, else there is a vertex not in $T$ which is adjacent to exactly one vertex in $T$, which contradicts the assumption that $S$ is a maximal 1-adjacent vertex sequence.

Proposition 5.7. For any graph $G, g_{1 \mathrm{a}}(\mathrm{G})$ equals the maximum order of an induced subtree in $G$.

Proof. For any graph $G$, Proposition 5.6 implies that $\operatorname{gr}_{1 \mathrm{a}}(\mathrm{G})$ is at most the maximum order of an induced subtree in $G$.

Let $T$ be an induced subtree of a graph $G$ having maximum order $r$. Let $T$ be rooted at a vertex $v_{1}$, and let $V=$ $\left\{v_{1}, v_{2}, \ldots, v_{r}\right\}$ be the $r$ vertices of $T$, listed in breadth-first order, as before. Let the maximum distance in $T$ from $v_{1}$ to a vertex in $T$ be $k$, that is, there exists at least one vertex $x \in V(T)$ such that $d_{T}\left(v_{1}, x\right)=k$. Let $D_{i}=\left\{v_{j} \mid d_{T}\left(v_{j}, v_{1}\right)=i\right\}$ be the set of vertices at distance $i$ from the root $v_{1}$ in $T$. Consider the sequence of sets $D_{1}, D_{2}, \ldots, D_{k}$. Form a sequence $S$ of vertices in $V(T)$ by listing $v_{1}$ first, followed by all vertices, in any order, in set $D_{1}$, then by all vertices in $D_{2}$, etc., until all vertices in $V(T)$ have been listed, and we have a sequence $S=\left(v_{1}, v_{2}, \ldots, v_{r}\right)$ of the $r$ vertices of $T$. Consider any vertex $v_{i}$ in this sequence. Now $v_{i}$ is adjacent to exactly one vertex in $\widehat{S}_{i-1}$, namely, its parent. Thus, $v_{i}$ qualifies to be listed next after the sequence $v_{1}, v_{2}, \ldots, v_{i-1}$. Thus, $S$ is a 1-adjacent vertex sequence, not only in $T$ but in $G$. Hence, $\operatorname{gr}_{1 \mathrm{a}}(\mathrm{T}) \geq \mathrm{r}$.

This proposition is interesting because of the complement $\bar{S}$ of $S$, in which every vertex must be adjacent to two or more vertices in $S$ or no vertices in $S$.

\subsection{Acyclic vertex sequences}

A sequence $S=\left(v_{1}, v_{2}, \ldots, v_{k}\right)$ of vertices in a graph $G$ is called an acyclic vertex sequence if for every vertex $v_{i}$, the induced subgraph $G\left[S_{i}\right]$ is acyclic. The maximum length of an acyclic vertex sequence is called the Grundy acyclic number and is denoted by $\operatorname{gr}_{\mathrm{ac}}(\mathrm{G})$.

Observation 5.2. For any graph $G$, the minimum length of a maximal acyclic vertex sequence equals the minimum order of a maximal induced forest in $G$.

Observation 5.3. For any graph $G, \mathrm{gr}_{\mathrm{ac}}(\mathrm{G})$ equals the maximum order of an induced forest in $G$.

\subsection{Other sequences}

We close with the following list which illustrates the wide variety of types of vertex sequences which can be defined and studied.

1. a different distance sequence $S=\left(v_{1}, v_{2}, \ldots, v_{k}\right)$ is a vertex sequence having the property that for every $v_{i}$, no vertex in $V \backslash \widehat{S}_{i}$ is equidistant to two vertices in $\widehat{S}_{i}$.

2. a greedy $k$-coloring sequence $S=\left(v_{1}, v_{2}, \ldots, v_{k}\right)$ is a vertex sequence, each vertex $v_{i}$ of which is properly $k$-colored in $G\left[\widehat{S}_{i}\right]$ (no previous neighbor in the sequence is assigned the same color and at most $k$ colors are assigned to the vertices in $\widehat{S}_{i}$ ) and the color assigned to every vertex in $\widehat{S}_{i}$ is the least color not assigned to any previous neighbor in $S_{i}$.

3. a cost effective sequence $S=\left(v_{1}, v_{2}, \ldots, v_{k}\right)$ is a vertex sequence having the property that for every $v_{i}$, every vertex $v_{j} \in \widehat{S}_{i}$ has at least as many neighbors in $V \backslash \widehat{S}_{i}$ as it has in $\widehat{S}_{i}$. 
4. a signed domination sequence $S=\left(v_{1}, v_{2}, \ldots, v_{k}\right)$ is a vertex sequence having the property that for every $v_{i}$, every vertex $v_{j} \in \widehat{S}_{i}$ has more neighbors in $V \backslash \widehat{S}_{i}$ than it has in $\widehat{S}_{i}$.

5. a restrained vertex sequence $S=\left(v_{1}, v_{2}, \ldots, v_{k}\right)$ is a vertex sequence having the property that for every $v_{i}$, every vertex in $V \backslash \widehat{S}_{i}$ has a neighbor in $V \backslash \widehat{S}_{i}$, that is, $G\left[V \backslash \widehat{S}_{i}\right]$ has no isolated vertices.

6. an almost perfect sequence $S=\left(v_{1}, v_{2}, \ldots, v_{k}\right)$ is a vertex sequence having the property that for every $v_{i}$, every vertex $v_{j} \in \widehat{S}_{i}$ either has no neighbors in $\widehat{S}_{i}$ or is adjacent to a vertex $v_{r} \in \widehat{S}_{i}$ such that $\left|N\left[v_{r}\right] \cap \widehat{S}_{i}\right|=1$.

7. a chordal graph sequence $S=\left(v_{1}, v_{2}, \ldots, v_{k}\right)$ is a vertex sequence having the property that for every vertex $v_{i}, G\left[N\left[v_{i}\right] \cap\right.$ $\left.\widehat{S}_{i}\right]$ is a complete subgraph, that is the neighbors of $v_{i}$ in $\widehat{S}_{i-1}$ form a complete subgraph.

\section{References}

[1] A. Aazami, Hardness Results and Approximation Algorithms for Some Problems on Graphs, PhD Thesis, University of Waterloo, Waterloo, 2008.

[2] A. Aazami, Domination in graphs with bounded propagation: algorithms, formulations and hardness results. J. Comb. Optim. 19 (2010) $429-456$.

[3] A. Aazami, M. D. Stilp, Approximation algorithms and hardness for domination with propagation, In: Proceedings of the 10th International Workshop on Approximation Algorithms for Combinatorial Optimization Problems, Lecture Notes in Comput. Sci. 4627, Springer, New York, 2007, pp. 1-15.

[4] A. Aazami, K. Stilp, Approximation algorithms and hardness for domination with propagation, SIAM J. Discrete Math. 23 (2009) $1382-1399$.

[5] S. Arumugam, S. T. Hedetniemi, S. M. Hedetniemi, L. Sathikala, S. Sudha, The covering chain of a graph, Util. Math. 98 (2015) 183-196.

[6] T. L. Baldwin, L. Mili, M. B. Boisen, R. Adapa, Power system observability with minimal phasor measurement placement, IEEE Trans. Power Syst. 8 (1993) 707-715.

[7] F. Barioli, W. Barrett, S. Butler, S. M. Cioaba, D. Cvetkovic, S. M. Fallat, C. Godsil, W. Haemers, L. Hogben, R. Mikkelson, S. Narayan, O. Pryporova, I. Sciriha, W. So, D. Stevanovic, H. van der Holst, K. Vander Meulen, A. Wangsness, Zero forcing sets and the minimum rank of graphs, Linear Algebra Appl. 428 (2008) 1628-1648.

[8] B. Brešar, C. Butjás, T. Gologranc, S. Klavžar, G. Košmrlj, B. Patkós, Z. Tuza, M. Vizer, Dominating sequences in grid-like and toroidal graphs, Electron. J. Combin. 23 (2016) Art\# 4.34.

[9] B. Brešar, C. Butjás, T. Gologranc, S. Klavžar, G. Košmrlj, B. Patkós, Z. Tuza, M. Vizer, Grundy dominating sequences and zero forcing sets, Discrete Optim. 26 (2017) 66-77.

[10] B. Brešar, T. Gologranc, T. Kos, Dominating sequences under atomic changes with applications in Sierpiński and interval graphs, Appl. Anal. Discrete Math. 10 (2016) 518-531.

[11] B. Brešar, T. Gologranc, M. Milanič, D. F. Rall, R. Rizzi, Dominating sequences in graphs, Discrete Math. 336 (2014) 22-36.

[12] B. Brešar, M. A. Henning, D. F. Rall, Total dominating sequences in graphs, Discrete Math. 339 (2016) 1665-1676.

[13] B. Brešar, S. Klavžar, D. F. Rall, Domination game and an imagination strategy, SIAM J. Discrete Math. 24 (2010) 979-991.

[14] B. Brešar, T. Kos, G. Nasini, P. Torres, Total dominating sequences in trees, split graphs, and under modular decomposition, Discrete Optim. 28 (2018) 16-30.

[15] I. Broere, E. Jonck, G. S. Domke, The induced path number of the Cartesian product of some graphs, Australas. J. Combin. 36 (2006) 3-12.

[16] D. J. Brueni, Minimal PMU Placement for Graph Observability: A Decomposition Approach, Masters thesis, Virginia Polytechnic Institute and State University, Blacksburg, 1993.

[17] D. J. Brueni, L. S. Heath, The PMU placement problem, SIAM J. Discrete Math. 19 (2005) 744-761.

[18] F. Buckley, F. Harary, On longest induced paths in graphs, Chinese Quart. J. Math. 3 (1988) 61-65.

[19] E. J. Cockayne, P. J. P. Grobler, S. T. Hedetniemi, A. A. McRae, What makes an irredundant set maximal?, J. Combin. Math. Combin. Comput. 25 (1997) 213-223.

[20] E. J. Cockayne, J. H. Hattingh, S. M. Hedetniemi, S. T. Hedetniemi, A. A. McRae, Using maximality and minimality conditions to construct inequality chains, Discrete Math. 176 (1997) 43-61.

[21] E. J. Cockayne, S. T. Hedetniemi, D. J. Miller, Properties of hereditary hypergraphs and middle graphs, Canad. Math. Bull. 21 (1978) $461-468$.

[22] J. H. Hattingh, O. A. Saleh, L. C. Van der Merwe, T. J. Walters, Nordhaus-Gaddum type results for the induced path number with relative complements in $K_{m, n}$, J. Combin. Math. Combin. Comput. 85 (2013) 365-377.

[23] T. W. Haynes, S. M. Hedetniemi, S. T. Hedetniemi, M. A. Henning, Domination in graphs applied to electric power networks, SIAM J. Discrete Math. 15 (2002) 519-529.

[24] T. W. Haynes, S. T. Hedetniemi, P. J. Slater, Fundamentals of Domination in Graphs, Marcel Dekker, New York, 1998.

[25] M. A. Henning, S. Klavžar, D. F. Rall, Total version of the domination game, Graphs Combin. 31 (2015) 1453-1462.

[26] T. Ishizeki, Y. Otachi, K. Yamazaki, An improved algorithm for the longest induced path problem on $k$-chordal graphs, Discrete Appl. Math. 156 (2008) 3057-3059.

[27] J. C.-H. Lin, Zero forcing number, Grundy domination number, and their variants, Linear Algebra Appl. 563 (2019) $240-254$. 